\title{
Lattice contributions to the anisotropic dielectric response of rare-earth langasites
}

\author{
L. Bergen $\odot,{ }^{1}$ L. Weymann,,${ }^{1}$ J. Wettstein, ${ }^{1}$ A. M. Kuzmenko, ${ }^{2}$ A. A. Mukhin, ${ }^{2}$ B. V. Mill, ${ }^{3}$ A. Pimenov,,${ }^{1}$ and E. Constable $\oplus^{1}$ \\ ${ }^{1}$ Institute of Solid State Physics, Vienna University of Technology, 1040 Vienna, Austria \\ ${ }^{2}$ Prokhorov General Physics Institute Russian Academy of Sciences, 119991 Moscow, Russia \\ ${ }^{3}$ Moscow State University, 119991 Moscow, Russia
}

(Received 2 March 2021; revised 5 May 2021; accepted 17 June 2021; published 30 July 2021)

\begin{abstract}
We investigate the phonon spectrum of pure and rare-earth substituted langasites. For electric fields oriented along the $c$ axis, a strong low-frequency phonon is observed, softening at lower temperature in all samples studied. The dielectric contribution and temperature dependence of this mode is the primary source of the large $[\varepsilon(0) \sim 100]$ static dielectric permittivity observed in these systems. Softening of this mode with increasing mass of the rare-earth substitutes further suggests that langasites are close to a structural instability at low temperatures. The application of a magnetic field to a diluted Ho-langasite sample reveals a shift in the strong absorption band at $\mathrm{THz}$ frequencies associated with this soft lattice mode, suggesting spin-lattice effects may also be at play in these systems.
\end{abstract}

DOI: 10.1103/PhysRevB.104.024106

\section{INTRODUCTION}

The presence of orthogonal threefold and twofold rotation axes in the $P 321$ space group sets the stage for a number of intriguing functional properties expressed across a range of real material compounds. A prime example is the diversity of phenomena exhibited by the langasite family, with parent chemical formula $\mathrm{La}_{3} \mathrm{Ga}_{5} \mathrm{SiO}_{14}$ (LGS) and $P 321$ space-group symmetry [1]. As shown in Fig. 1, langasites are constructed by two alternating layers, each with threefold symmetry, stacked along the $c$ direction. The first layer consists of $\mathrm{La}^{3+}$ decahedra ( $3 e$ sites with $C_{2}$ symmetry) and $\mathrm{Ga}^{3+}$ octahedra (1a sites with $D_{3}$ symmetry). The second layer consists of $\mathrm{Ga}^{3+}$ ( $3 f$ sites with $C_{2}$ symmetry) and $\mathrm{Si}^{4+} / \mathrm{Ga}^{3+}(2 d$ sites with $C_{3}$ symmetry) tetrahedra. Naturally the oxygen ions are coordinated at the vertices of each polyhedra.

As insulators lacking an inversion symmetry, the langasites have long been of interest for their piezoelectric properties [2-4]. While in equilibrium the orthogonal rotation axes forbid the presence of polar order, the loss of either rotation symmetry easily promotes one. In the case of langasites, a uniaxial pressure compressing the $a$ axis suppresses the threefold symmetry inducing $a$-axis polarization [5]. Significant voids between the $\mathrm{La}^{3+}$ decahedra and $\mathrm{Ga}^{3+}$ octahedra, coupled with their threefold arrangement in the $a b$ plane, make them particularly susceptible to $a$-axis stress [4]. Indeed, the mechanical coupling coefficient of langasite significantly exceeds that of quartz [6]. This, along with its structural stability over a broad temperature range, make langasite particularly attractive for device applications utilizing piezoelectric sensing [7].

Published by the American Physical Society under the terms of the Creative Commons Attribution 4.0 International license. Further distribution of this work must maintain attribution to the author(s) and the published article's title, journal citation, and DOI.
Beyond piezoelectricity, the so-called rare-earth langasites have also garnered interest for their intriguing magnetic properties [8]. In such systems, the $\mathrm{La}^{3+}$ sites are substituted with heavier rare-earth elements that feature magnetic ground states. Again, the threefold symmetry plays its part, this time promoting magnetic frustration, with the triangular arrangement of magnetic ions forming the familiar (although distorted) Kagome lattice in the $a b$ plane. In the example of Nd langasite (Nd-LGS), despite considerable antiferromagnetic correlations, no long-range magnetic order develops even down to $\sim 50 \mathrm{mK}[9,10]$. Rather, due to competition imposed by the threefold geometric structure, a single-ion quantum process emerges promoting a fluctuating spin state reminiscent of a quantum spin liquid [11].

In the closely related $\mathrm{Fe}$ langasites (e.g., $\mathrm{Ba}_{3} \mathrm{TaFe}_{3} \mathrm{Si}_{2} \mathrm{O}_{14}$ ), magnetic $S=5 / 2 \mathrm{Fe}^{3+}$ ions are situated on the $\mathrm{Ga}^{3+} 3 f$ sites, characterized by a network of isolated triangles [12-14]. While the threefold symmetry of the triangular arrangement also promotes frustration, here it is ultimately lifted by chiral next-nearest-neighbor exchange pathways supported by the twofold symmetry normal to the $c$ axis $[12,13]$. This, in combination with single-ion anisotropy and the Dzyaloshinskii-Moriya interaction, induces a novel spiral magnetic order coupling the broken time-reversal symmetry to the two rotational symmetries $[12,15]$. No wonder then that the Fe langasites express a diversity of both static and dynamic magnetoelectric effects with the application of external magnetic fields able to induce polarization states by breaking the spacial-rotation symmetries [16-18].

In a more recent example, magnetoelectric effects are also observed even in the absence of magnetic order. Recently, a novel combination of linear and highly nonlinear magnetoelectric responses were observed in the disordered paramagnetic state of holmium-doped rare earth langasite $\mathrm{Ho}_{x} \mathrm{La}_{3-x} \mathrm{Ga}_{5} \mathrm{SiO}_{14}$ [19]. Here it is believed that an interplay between the global $C_{3}$ symmetry of the $a b$ plane and the local $C_{2}$ symmetry of the magnetic $\mathrm{Ho}^{3+}$ sites is behind the novel magneto-electric response. While the precise details are 
still under investigation, it is likely that spin-lattice coupling, and the complex distribution of local $\mathrm{Ho}^{3+}$ Ising axes, due to different coordinations of the $\mathrm{Si}^{4+} / \mathrm{Ga}^{3+} 2 d$ sites, play an important role.

A key property that underlines the diverse behavior of langasites, particularly concerning their functional applications, is the response to external electric fields. Dielectrically, the langasites display strong anisotropy, with the reported dielectric permittivity of the threefold $(c)$ axis being several times larger than that of the twofold ( $a$ and $b^{*}$ ) axes [1]. At first glance this too is linked to the different ways the orthogonal $C_{3}$ and $C_{2}$ rotation symmetries behave optically. However, despite significant research on langasites, the microscopic details and lattice dynamics behind this dielectric anisotropy remain to be fully explored.

In this article we attempt this exploration by investigating the polar lattice dynamics of pure and rare-earth langasite samples using Fourier transform infrared (FTIR) reflection spectroscopy. The results characterize the lattice dynamics as a function of temperature and rare-earth element. We find that for radiation polarized along the $c$ (threefold) axis, the dielectric response is dominated by the large dipolar moment of an anomalous low-frequency phonon displaying soft-mode behavior and splitting at low temperatures. The results are discussed in the context of the space-group symmetry $\mathrm{Si}^{4+} / \mathrm{Ga}^{3+}$ coordination, and indicate a potential structural instability to a close-lying polar phase.

The anomalous low-frequency lattice mode is also addressed in the context of spin-lattice coupling and the role it may play in the frustrated ground states of the rare-earth langasites, as well as in the irregular magnetoelectric response of Ho-doped langasite. In support of this, we also perform complementary magneto-optical $\mathrm{THz}$ measurements revealing a magnetic field dependence in the spectral signature of the anomalous low-frequency phonon in Ho-doped langasite. This result, in particular, highlights a potential hybridization between the lattice dynamics and low-energy crystal electric field levels in these systems.

\section{EXPERIMENTAL DETAILS}

In total, four single crystal langasite samples were investigated. A commercially available pure $\mathrm{La}_{3} \mathrm{Ga}_{5} \mathrm{SiO}_{14}$ (LGS) sample, along with three rare-earth substituted langasites grown using the Czochralski technique in Moscow State University [1]. These included Nd-langasite $\mathrm{Nd}_{3} \mathrm{Ga}_{5} \mathrm{SiO}_{14}$ (Nd-LGS), Pr-langasite $\mathrm{Pr}_{3} \mathrm{Ga}_{5} \mathrm{SiO}_{14}$ (Pr-LGS), and partially substituted Ho-langasite $\left(\mathrm{Ho}_{x} \mathrm{La}_{1-x}\right)_{3} \mathrm{Ga}_{5} \mathrm{SiO}_{14}$ with $x=0.015 \pm 0.002$ (Ho-LGS). Due to the substantial difference in ionic radii of $\mathrm{La}^{3+}$ and $\mathrm{Ho}^{3+}$, stable crystals of fully substituted Ho-LGS are not currently available. All single crystal samples were polished and cut to a typical size of $\sim 7 \times 7 \times 1 \mathrm{~mm}^{3}$ with surfaces normal to the $a$ axis ( $a$ cut) and the $c$ axis ( $c$ cut).

The infrared reflectance measurements were performed using a Bruker vertex 80v FTIR spectrometer. Room temperature measurements were performed between 20 and $3000 \mathrm{~cm}^{-1}$ using polarized radiation from a Hg lamp and globar source. The polarization of incident radiation was controlled by a rotating wire-grid polarizer positioned before the sample. Radiation between $20-700 \mathrm{~cm}^{-1}$ was detected by a liquid-He-cooled silicon bolometer. Radiation between $700-3000 \mathrm{~cm}^{-1}$ was detected using a liquid- $\mathrm{N}_{2}$-cooled MCT detector. The samples were mounted behind a $4 \mathrm{~mm}$ aperture masked by silicon-carbide abrasive paper, which gives almost no background reflection over the spectral range investigated. A gold mirror replacing the sample was used as a reference. Low temperature measurements between 10-300 K were performed in an Oxford instruments He-flow cryostat fitted with polyethylene windows. Due to the transmissive properties of the window materials, the low temperature reflectance was only investigated in the frequency range of $650 \mathrm{~cm}^{-1}$, focusing on the infrared phonons.

To obtain the dielectric response as a function of frequency, $\varepsilon(v)=\varepsilon_{1}(v)+i \varepsilon_{2}(v)$, we assume near-normal incidence relating the complex reflectivity $\sqrt{R} e^{i \varphi}$ to the complex dielectric response with the form

$$
\varepsilon(v)=\left(\frac{1+\sqrt{R} e^{i \varphi}}{1-\sqrt{R} e^{i \varphi}}\right)^{2} .
$$

Here the phase shift $\varphi(v)$ is calculated using a KramersKronig (KK) transformation of the experimentally obtained reflectance data $R(v)$. The KK transform assumes a reflectivity stretching from zero to infinite frequency. Therefore, to approximate the reflectivity extrapolated to zero, the lowest frequency data is fit using the RefFIT software package [20] providing a model Lorentzian profile of the lowest frequency lattice vibration that we extend to zero frequency. For the high-frequency approximation, we found that a simple constant reflectivity value extrapolated from the value at 3000 up to $15000 \mathrm{~cm}^{-1}$ gave an adequate approximation for our investigations focused on the far infrared. For the low-temperature spectra measured within the cryostat (where measurements are limited to below $650 \mathrm{~cm}^{-1}$ ), we use the room temperature scan in the range between 600 and $3000 \mathrm{~cm}^{-1}$. We found that the lattice dynamics in this frequency range do not alter much at low temperatures and that this procedure does not significantly affect our observations in the far-infrared range below $650 \mathrm{~cm}^{-1}$. With the dielectric response extracted by the KK transform procedure, we then perform fits using the Lorentz oscillator model,

$$
\varepsilon(\omega)=\varepsilon_{\infty}+\sum_{i} \frac{\Delta \varepsilon_{i} \omega_{i}^{2}}{\omega_{i}^{2}-\omega^{2}-i \omega \gamma_{i}} .
$$

Here $\omega_{i}$ is the resonance frequency, $\gamma_{i}$ is the damping, $\Delta \varepsilon$ is the dielectric contribution, $\varepsilon_{\infty}$ is the high-frequency dielectric permittivity, and $\omega=2 \pi v$ is the angular frequency. Uncertainties for these parameters are determined by the difference between fits when incorporating a variance of 5\% in the measured reflectivity, which we take as a modest estimation for the variance in the measurement due to sample and reference alignment.

The results were then compared to low-frequency capacitive measurements performed at $v=10 \mathrm{kHz}$ using an Alpha-Analyzer combined with a Quantum Design Physical Property Measurement System (PPMS). Silver paint contacts were applied to the opposite sides of the plane-parallel sample approximating a parallel plate capacitor. Attempts to apply static magnetic field up to $14 \mathrm{~T}$ did not lead to measurable magnetocapacitance effects. 

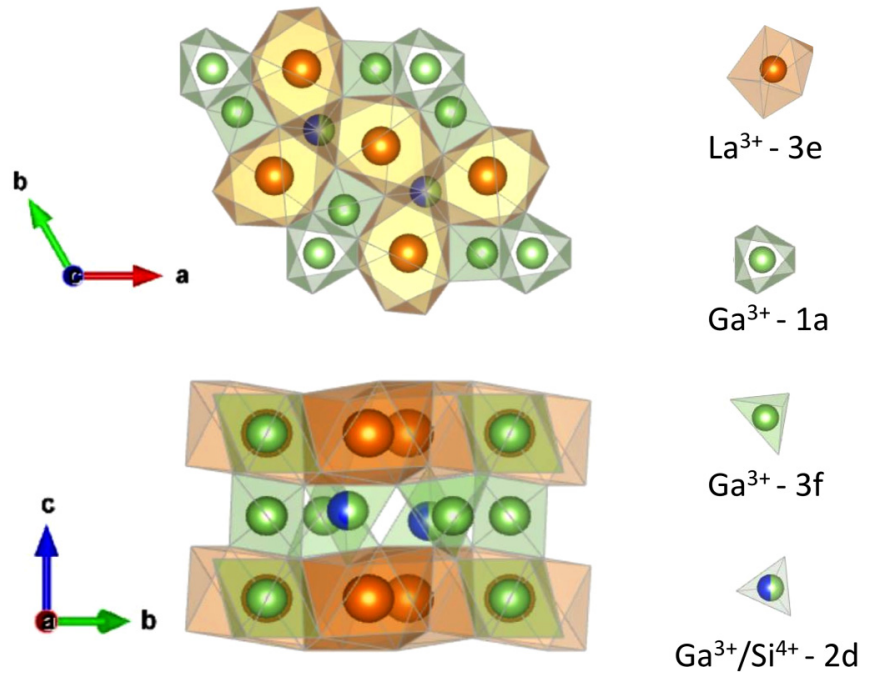

FIG. 1. Crystal structure of langasite. The geometry of the different sites is labeled on the right along with the ions and Wycoff positions.

Complementary magneto-optical $\mathrm{THz}$ measurements were performed on the Ho-LGS sample using a Mach-Zehnder interferometer with backwards wave oscillator sources and $\mathrm{Si}$ bolometer detector [21]. The sample was mounted in the variable temperature insert of an $8 \mathrm{~T}$ Oxford split-ring superconducting magnet.

\section{RESULTS}

Typical reflectance spectra of the LGS sample are shown in Fig. 2. For light polarized parallel to the threefold $c$ axis [e\|c Fig. 2(a)], the spectra are dominated by a strong, low-

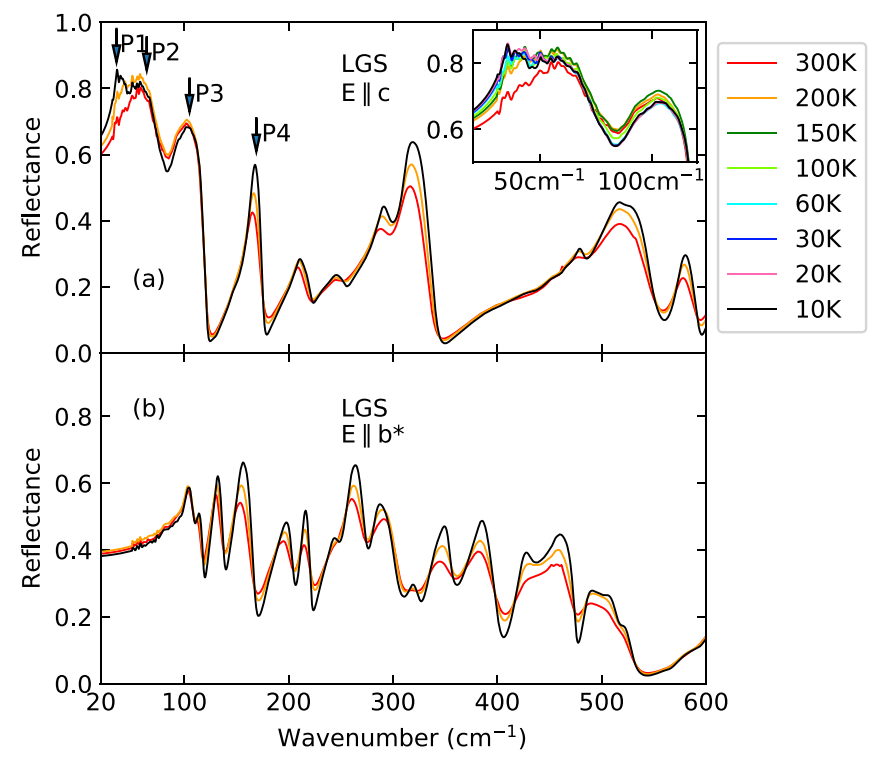

FIG. 2. Reflectance of LGS parallel (a) and perpendicular (b) to the polar $c$ axis at three temperatures as indicated. The arrows in the top panel indicate the four lowest frequency lattice modes in $c$ direction labeled P1-P4. Results for the full spectral range out to $2000 \mathrm{~cm}^{-1}$ can be found in the Supplemental Material [22].

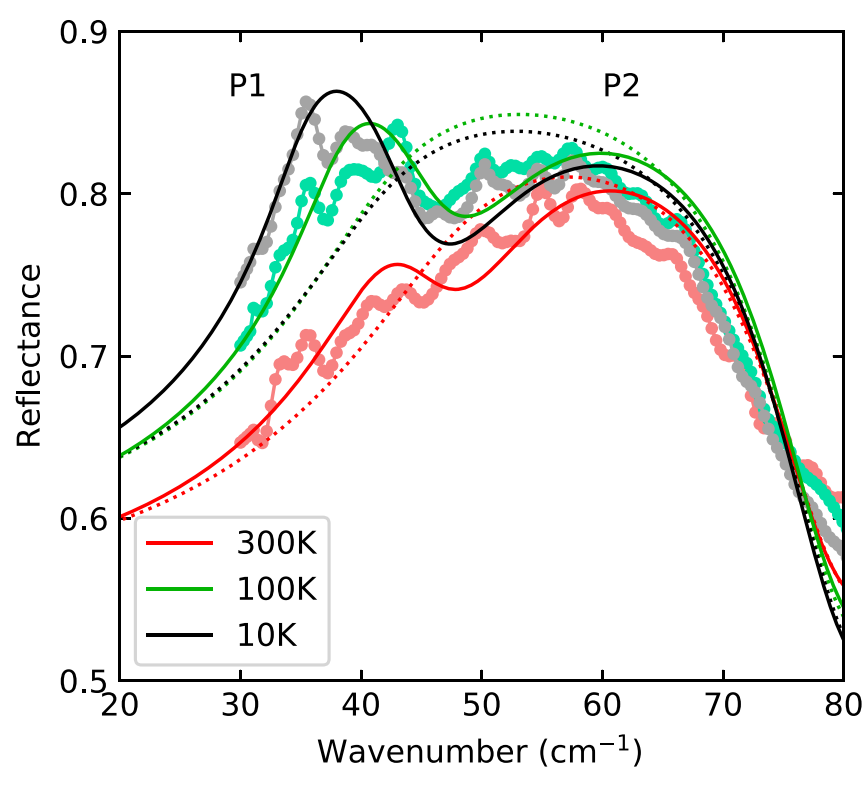

FIG. 3. Reflectance of LGS parallel to the $c$ axis at three temperatures as indicated. The data points represent the raw measurements, solid lines represent the fit with two oscillators, while dashed lines show fits for one oscillator.

frequency phonon around $40 \mathrm{~cm}^{-1}$. In the $e \| b^{*}$ geometry [twofold axis, Fig. 2(b)], all the lattice vibrations are much weaker and appear above $100 \mathrm{~cm}^{-1}$.

Notably, the profile of the phonon spectra for $e \| b^{*}$ remains largely unchanged over the full temperature range investigated. This is also true of the phonon spectra for $e \| c$ above $100 \mathrm{~cm}^{-1}$. Only the strong low-frequency phonon seems to show a significant temperature dependence. As highlighted in Fig. 3, below $\sim 200 \mathrm{~K}$, this phonon also begins to develop a finer structure.

As evidence for this splitting, we show the results of our fitting procedure using one and two oscillators to fit these features in Fig. 3. Here we see, particularly at low temperatures, the two phonon model provides a much better fit to the data. As the low-frequency phonons for $e \| c$ are most relevant to the static dielectric properties, we label them P1 to P4 and continue our analysis with a focus on them.

The extracted dielectric response for $e \| c$ is summarized in Fig. 4. At low temperatures, P1 sharpens and softens substantially with the separation between $\mathrm{P} 1$ and $\mathrm{P} 2$ now clearly seen in $\varepsilon_{2}$. Higher-frequency phonons for both $e \| c$ and $e \| b^{*}$ show typical sharpening as the lattice contracts with decreasing temperature, but otherwise remain stable. In total, we observe 17 modes along the $c$ direction (P1 and $\mathrm{P} 2$ counted individually) and 22 modes in the $a b$ plane.

Figure 5 shows the resonance frequency and the dielectric contribution of the first four phonons along the $c$ axis as a function of temperature obtained using the fitting procedure described above. Here we confirm that the frequency positions and dielectric contributions of $\mathrm{P} 3$ and $\mathrm{P} 4$ remain mostly stable over the investigated temperature range, while the two lowfrequency phonons show substantial temperature dependence. Specifically, the frequency of P1 decreases by about $15 \%$ upon cooling to $10 \mathrm{~K}$ providing an indication of structural instabil- 


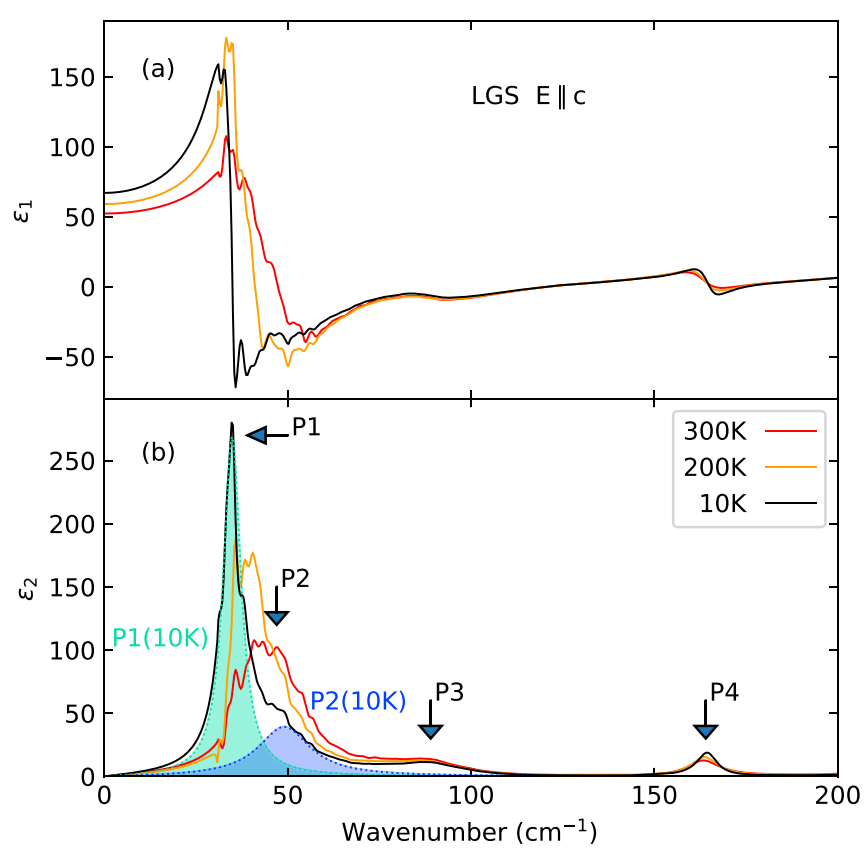

FIG. 4. Real (a) and imaginary part (b) of the dielectric permittivity $\varepsilon^{*}=\varepsilon_{1}+i \varepsilon_{2}$ for LGS at 10,200 , and $300 \mathrm{~K}$ for $e \| c$. Results for the full spectral range out to $600 \mathrm{~cm}^{-1}$ and for $e \| b^{*}$ can be found in the Supplemental Material [22].

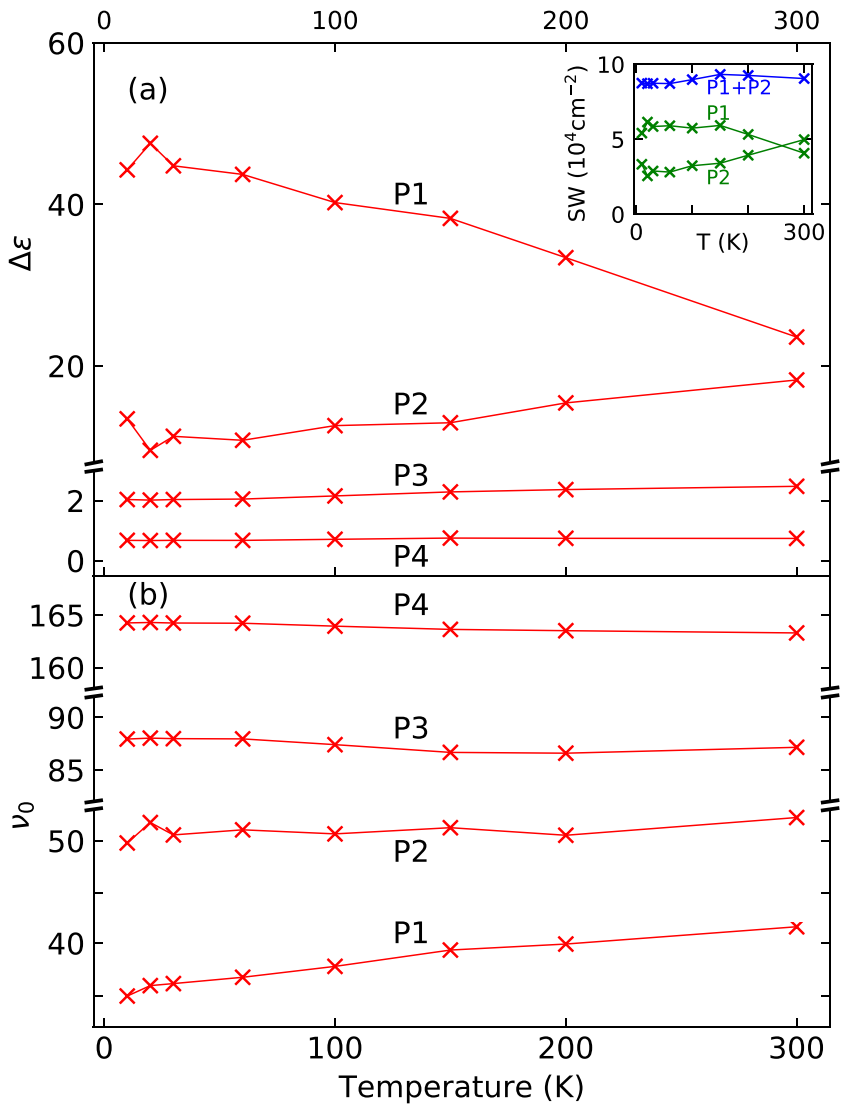

FIG. 5. (a) Dielectric contribution $\Delta \varepsilon$ of the first four oscillators with $e \| c$, demonstrating the dominant contribution of the P1 and P2 phonons. (b) Peak positions of the first four phonons obtained from the fits to $\varepsilon_{2}(\omega)$. The inset in (a) shows the spectral weight of two lowest phonons.

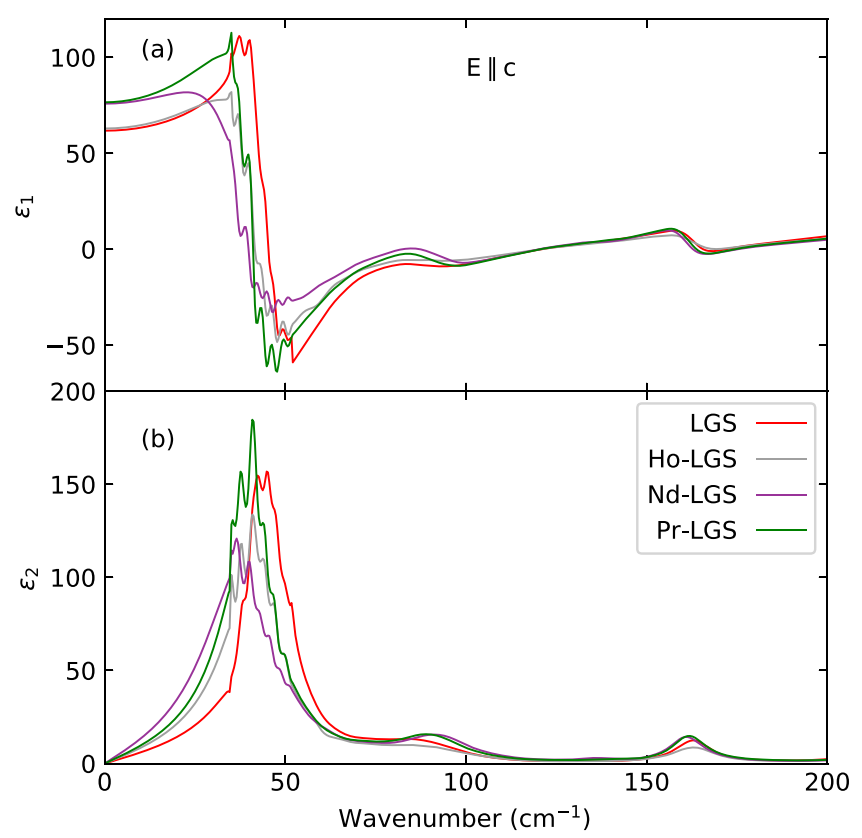

FIG. 6. Comparison of (a) real and (b) imaginary dielectric permittivity of the four different langasite samples at room temperature along the $c$ axis in the low-frequency range.

ity. For P2, a more subtle softening at lower temperature is also observable.

Notably, it is also $\mathrm{P} 1$ and $\mathrm{P} 2$ that exhibit the largest dielectric contributions $\left(\Delta \varepsilon_{1}+\Delta \varepsilon_{1} \gtrsim 50\right)$, dominating the other phonons and being solely responsible for the high value of the static dielectric permittivity along the $c$ axis. The strength of P1 increases upon cooling, which correlates with the softening of the resonance frequency. Simultaneously, the contribution of P2 decreases demonstrating a transfer of spectral weight (SW $\sim \Delta \varepsilon v_{0}^{2}$ ) to $\mathrm{P} 1$. This transfer is confirmed in the inset of Fig. 5, where we show that the sum of the spectral weights of P1 and P2 remains reasonably constant over the whole temperature range.

To investigate the influence of rare-earth substitution in the lattice dynamics of langasites, we have measured the reflectivity of Nd-LGS, Pr-LGS, and Ho-LGS samples shown in Fig. 6. In this plot, only the room temperature results are presented, which were obtained without the cryostat. In general, the overall shape of the dielectric response is similar for all samples in both polarizations. Notably, the anomalous phonon behavior along the $c$ axis is found in all samples investigated. The strength and frequency of these modes display a clear dependence on the rare-earth substitution. While there are indeed some differences in the strength and frequencies of many of the other phonons, these changes are much more subtle. There are however, a few of small excitations that seem to be sample dependent, with a weak shoulder around $120 \mathrm{~cm}^{-1}$ for $e \| b^{*}$ only being visible in the LGS and in Ho-LGS spectra.

Showcasing the most striking differences with rare earth substitution, we now turn our attention to the dynamics of the lowest-frequency phonons for $e \| c$. As is evident in Fig. 3 $\mathrm{P} 1$ and $\mathrm{P} 2$ are difficult to distinguish close to room temperature. Therefore, here, a spectral profile incorporating just a single oscillator is used. This then represents an average 


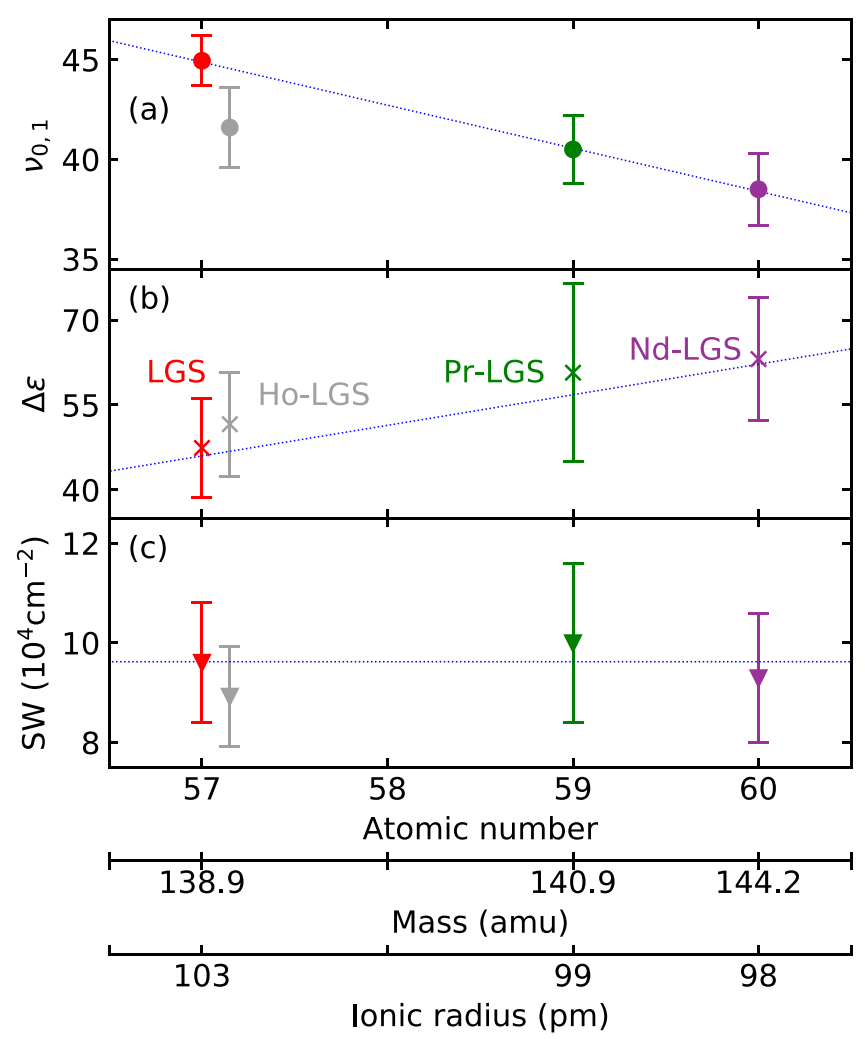

FIG. 7. Parameters of the lowest-frequency phonon as function of atomic number for different rare-earth langasites. For the mixed Ho-LGS a weighted effective number has been utilized. (a) Resonance frequency, (b) dielectric contribution, and (c) spectral weight $\mathrm{SW}=\Delta \varepsilon v_{0,1}^{2}$. A linear trend in $\nu_{0,1}$ and in $\Delta \varepsilon$ for increasing atomic number is given by thin dotted lines. This behavior leads to approximately constant spectral weight of all langasites (horizontal line in the bottom panel). Additional axes for the masses and atomic radii are given for reference.

of the low-frequency phonon dynamics for the two modes. An interesting behavior of these modes is observed when plotting the resonance frequency and dielectric contribution as a function of the atomic number of the rare-earth element. As demonstrated in Fig. 7, a clear linear trend emerges showing a tendency towards a polar lattice instability $\left(v_{0} \rightarrow 0, \epsilon \rightarrow \infty\right)$ for larger atomic numbers. Due to the effect of lanthanide contraction with increasing atomic number (and increasing mass) this relationship is also linked to the decreasing ionic radius of the ions at the $\mathrm{La}^{3+}$ sites. Notably this correlation does not monotonically follow the increase in atomic mass.

As can be seen in the Ho-LGS sample, despite only a small concentration of Ho atoms, a significant shift in the lowfrequency phonon is observed. With an ionic radius of $90 \mathrm{pm}$, Ho is the smallest rare-earth element of those investigated. Extrapolating the trend set by the other samples, we could expect a shift of $21.6 \mathrm{~cm}^{-1}$ relative to LGS for a pure Ho-LGS sample. In order to position the results of the Ho-LGS sample in this chart, we have modified its atomic number by adding a value consistent to $1.5 \%$ Ho concentration to that of $\mathrm{La}$. This places the observation inline with the trend observed from the other compounds. Evidently even a very small amount of Ho causes significant distortion relative to the host LGS structure along the $c$ axis.

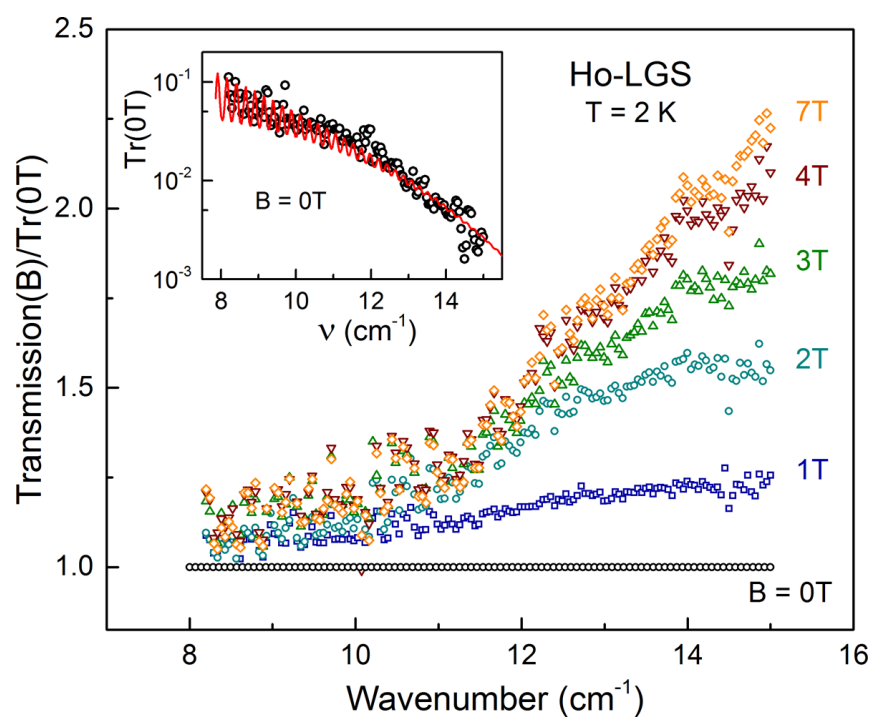

FIG. 8. Magneto-optical THz transmission measurements of HoLGS for electric field of light parallel to the $c$ axis. The inset shows the transmission spectrum at $0 \mathrm{~T}$ with a fit incorporating a single Lorentz oscillator (solid red curve). The main panel shows the relative change in transmission as magnetic field is applied along the $c$ axis of the sample in Voigt geometry.

In order to probe the spectral response below $\mathrm{P} 1$ and $\mathrm{P} 2$, we have performed $\mathrm{THz}$ transmission measurements on the Ho-LGS sample, as shown in the inset of Fig. 8. Here we observe the low-frequency shoulder of a strong absorption band consistent with the lowest phonons. The transmission at low frequencies is basically determined by the imaginary part of Eq. (2) that can be approximated as

$$
\operatorname{Im}[\varepsilon(\omega)] \approx \omega \sum_{i} \frac{\Delta \varepsilon_{i} \gamma_{i}}{\omega_{i}^{2}}
$$

and can be reduced to a response of a single Lorentzian with renormalized parameters. Due to the $\omega_{i}^{2}$ term in the denominator, the low-frequency absorption is dominated by the two lowest phonons P1 and P2. Although the mode parameters cannot be extracted from the terahertz transmission, the fit in the inset of Fig. 8 is consistent with the data obtained from the reflectance (Fig. 5).

A rather novel transmission response is observed when applying an external magnetic field to the sample along the $c$ axis in the Voigt geometry. As evident in Fig. 8, the transmission in this region increases with increasing magnetic field. This can be interpreted as a hardening or narrowing of the absorption band associated with $\mathrm{P} 1$. The process can be seen to saturate above $\sim 4 \mathrm{~T}$, which is in agreement with the saturated magnetic moments in the Ho-LGS sample [19]. Such behavior is indeed peculiar for a purely dielectric phonon and suggests the magnetoelectric coupling previously observed in this system [19] could result from hybridized spin-lattice dynamics.

Finally, we compare the static dielectric permittivity $\varepsilon(0)$ measured directly using the PPMS and Alpha-Analyzer at $10 \mathrm{kHz}$ with that obtained by summing the contributions of the lattice vibrations in the infrared experiment. Figure 9 presents a summary of these data along the crystallographic $c$ direction for the four langasites. In the case of LGS, the data from Mill 


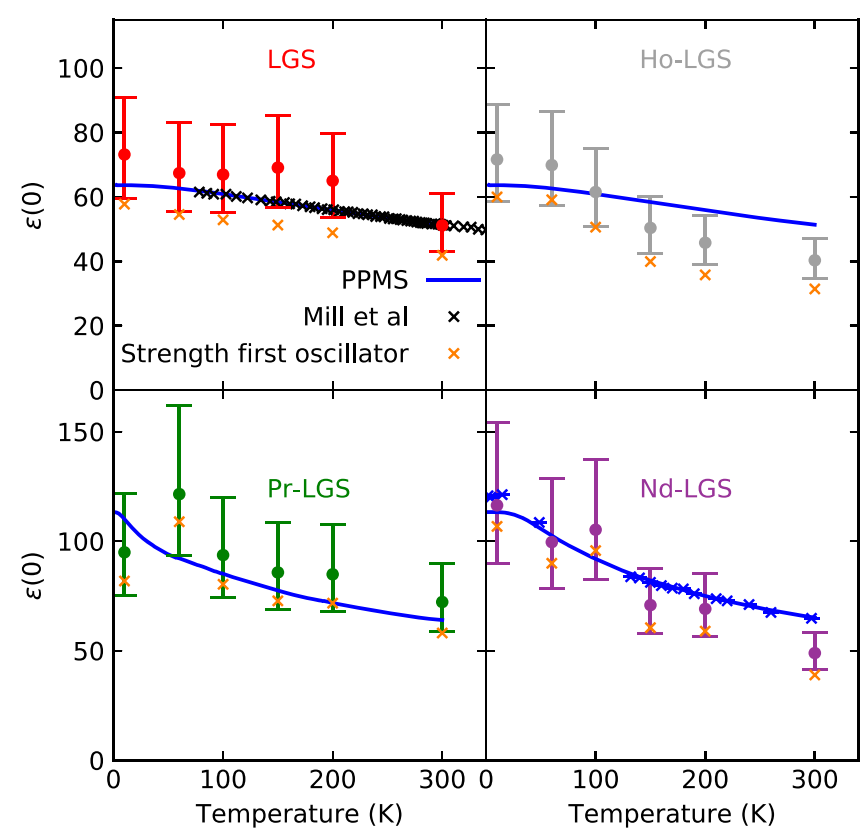

FIG. 9. Comparison of static dielectric permittivity of all four investigated langasite crystals. Filled circles represent the sum of dielectric contributions of all phonons obtained from the fit to the far-infrared spectra. The orange crosses represent the summed contributions from the two lowest-frequency phonons P1 and P2. The values of $\varepsilon_{0}$ were measured at a frequency of $v=10 \mathrm{kHz}$. In the LGS plot (top left), the static permittivity agrees closely with that of Mill et al. [1] (black crosses), in the Nd-LGS blue crosses on top of the PPMS data show additional measurements with a backward-wave oscillator performed at $3 \mathrm{~cm}^{-1}$.

et al. [1] are compared with our results for Ho-LGS showing a nice overlap. For comparison, the summed contribution from the low-frequency modes $(\mathrm{P} 1+\mathrm{P} 2)$ is plotted as open triangles. This highlights that the low-frequency phonons are mostly responsible for the large static dielectric permittivity along the $c$ axis and for the observed increase towards low temperatures.

\section{DISCUSSION}

Factor group analysis gives the following representation for lattice modes in the P321 langasite structure:

$$
\Gamma=11 A_{1}^{(\mathrm{R})}+A_{2}^{(\mathrm{A})}+13 A_{2}^{(\mathrm{IR})}+E^{(\mathrm{A})}+24 E^{(\mathrm{IR}+\mathrm{R})},
$$

where the superscripts designate acoustic (A), Raman (R), or infrared (IR) actives modes. Our observation of 39 excitations between $e \| c$ and $e \| b^{*}$ are in close agreement to the expected 37 infrared active modes predicted by group theory. The additional modes are possibly a result of degenerate modes appearing in both polarizations. However, we also expect that since we observe mode splitting for P1 and P2 at lower temperatures, the two extra modes could be a result of localized disorder across the shared $\mathrm{Si} / \mathrm{Ge}$ sites.

Recent $a b$ initio calculations performed on the closely related rare-earth langasites with chemical formula $\mathrm{Ln}_{3} \mathrm{CrGe}_{3} \mathrm{Be}_{2} \mathrm{O}_{14}(\mathrm{Ln}=\mathrm{La}, \mathrm{Pr}, \mathrm{Nd})$ indicate that the lanthanide elements contribute dominantly to the lower frequency vibrational modes [23,24]. At the same time, Raman spectra of LGS show that the first significant Raman active modes appear above $100 \mathrm{~cm}^{-1}$. For these reasons, and since our observations show that the lowest-frequency phonons have a strong dependence on rare-earth substitution, we can confidently assign the anomalous phonon modes designated $\mathrm{P} 1$ and $\mathrm{P} 2$ to $A_{2}$ dipolar oscillations of the $3 e$ (lanthanide) sites.

Based on this assignment, we can now begin to understand the origin of the dielectric anisotropy in LGS and its rareearth counterparts. First, we should highlight that while the measurements performed in the $e \| c$ geometry refer to the electric field polarized along the threefold rotation axis, these measurements are actually probing the $C_{2}$ symmetry, which transforms along this axis. At the same time, measurements performed in the $e \| b^{*}$ geometry (along the twofold axis) probe the $C_{3}$ symmetry. Therefore, dipole moments excited by the IR radiation in the $e \| b^{*}$ geometry are naturally balanced by their neighboring $\pm 120^{\circ}$ counterparts. This is similar to how the threefold symmetry promotes frustration of magnetic dipoles. Thus we naturally expect dipolar excitations in this plane to be suppressed and pushed to higher energies, as is observed.

On the other hand, for $e \| c$, dipoles freely add up along the twofold symmetry. Thus, whereas the large potential voids of the $\mathrm{La}^{3+}$ decahedra facilitate piezoelectricity along the $a$ axis under compression, here they facilitate large dipole moments along the $c$ axis under an electric field. This is also consistent with the observations of an increase in the phonon strength and softening of its frequency following rare-earth substitution. When heavier rare-earth elements are introduced with smaller atomic radii, the lattice naturally contracts. However, the contraction along the $c$ axis is only minor, with a change of $<0.1 \%$ between LGS and Nd-LGS despite a difference of $\sim 5 \%$ in atomic radii between $\mathrm{La}$ and $\mathrm{Nd}[4,5]$. Thus, with a smaller atomic radii but similar lattice dimensions, the vibrational displacement of the rare-earth site is allowed to increase, while the local restoring forces are reduced. This in turn lowers the energy of phonons associated with these sites while also supporting a larger electric dipole moment along the $c$ axis.

Lattice contraction in langasites is also observed as the temperature is lowered [25]. This causes most of the lattice excitations to sharpen in the spectra. The lowest energy phonon on the other hand, softens considerably. This behavior is likely tied to the asymmetric electron densities that surround the La ions [26]. If these asymmetries are enhanced at lower temperatures, there would be a predisposition for the heavy lanthanide elements to offset along the $c$ axis breaking the twofold symmetry. Similar ideas have been expressed following infrared and Raman studies on the Fe langasites [27]. While the softening is incomplete, it does suggest that a polar $P 3$ phase is tantalizing close and potentially accessible with the right chemical substitution and applied external electric field. Indeed, as shown in Fig. 10, the mass of the rare earth element affects the softening considerably. Here the temperature dependence of the frequency squared exhibits the classic linear soft-mode behavior indicating a monotonic reduction in the local restoring forces of the mode. Increasing the mass of the rare-earth ions moves the intercept at zero frequency to 


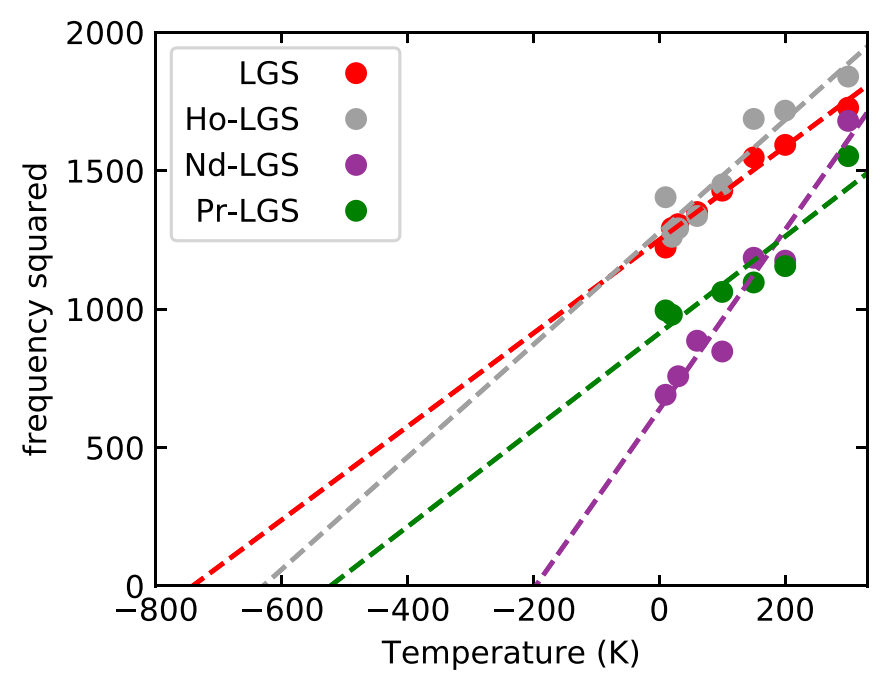

FIG. 10. Overview of the squared frequencies of the lowest oscillator from the fits. Dashed lines are linear extrapolations. These fits intercept the zero line at different temperature, increasing with atomic number.

higher temperatures, potentially approaching a positive value for even heavier rare-earth langasites.

One of the more interesting features of the soft-mode dynamics is its splitting at lower temperature. Since the shared spectral weight of $\mathrm{P} 1$ and $\mathrm{P} 2$ remains constant over the change in temperature, we can be confident that both excitations arise from similar dynamics relating to the lanthanide sites.

A distinguishing property of the langasites is the shared $50 \%$ occupation of $\mathrm{Si}^{4+}$ and $\mathrm{Ga}^{3+}$ at the $2 d$ Wycoff positions. In the local environment of the lanthanide sites, this means there can be up to nine nonequivalent coordinations of the $\mathrm{Si}^{4+}$ and $\mathrm{Ga}^{3+}$ ions. This distribution of local environments around the lanthanide ions would result in different lattice dynamics for each case. Our results suggest that of these different coordinations, two unique vibration dynamics emerge. One that remains largely stable at low temperatures (P2), and one that softens considerably (P1).

Finally, it is interesting to consider how these anomalous lattice dynamics may affect the local environment of magnetic ions in the rare-earth examples that express novel frustrated behavior. In the case of Nd-LGS, the magnetic susceptibility is seen to depart from the high-temperature Curie-Weiss behavior already between $100-300 \mathrm{~K}[8,11]$. This is typically a sign of a strong exchange-driven frustration. However, as studies performed on diluted samples of Nd-LGS demonstrate an identical temperature dependence of the magnetic susceptibility to the pure compound [11], this deviation may not be linked to exchange driven frustration at all. Rather, its correlation with the soft-mode behavior and splitting of the rare-earth lattice dynamics, as well as the first excited crystal electric field (CEF) level of $\mathrm{Nd}^{3+}$ falling at $\sim 55 \mathrm{~cm}^{-1}$ [28] (in close proximity to $\mathrm{P} 1$ ), suggests that spin-lattice dynamics may indeed be at play in the observations of anomalous magnetic susceptibility in Nd-LGS. Since $\mathrm{Nd}^{3+}$ is a Kramers ion, any coupling between phonons and CEF states would not be expected to break the degeneracy of any magnetic doublet states. Rather, only a shift in their respective energies might occur, which would be enough to modify the mean-field behavior as observed.

In non-Kramers ions such as $\mathrm{Ho}^{3+}$, the doublets are not protected. Thus it is possible that similar spin-lattice effects in Ho-LGS contribute to the complex shift between linear and nonlinear magnetoelectric behavior as observed over a broad temperature range in Ho-LGS [19]. Our observations of magnetic field dependent phonon dynamics in Ho-LGS are a strong indication of this behavior. In Ho-LGS a CEF level is expected at $30-50 \mathrm{~cm}^{-1}$ in the vicinity of $\mathrm{P} 1$ and $\mathrm{P} 2$ [19]. Depending on the global transformation of the CEF symmetry, it is possible that a vibronic coupling exists between the phonons and CEF level, driving novel spin-lattice effects in this system. In this respect we also highlight the potential for interesting spin-lattice behavior in other non-Kramers rare-earth langasites such as Tb-LGS, as is exhibited in its frustrated rare-earth pyrochlore counterpart [29].

\section{CONCLUSIONS}

Pure and rare-earth substituted crystals of the langasite $\left(\mathrm{La}_{3} \mathrm{Ga}_{5} \mathrm{SiO}_{14}\right)$ family were investigated by FTIR reflection and $\mathrm{THz}$ magneto-optical spectroscopy, and compared to capacitive results approaching the static response. In all compounds an anomalous strong low-frequency phonon $\left(\sim 40 \mathrm{~cm}^{-1}\right)$ can be observed for $e \| c$, which correlates well with the high dielectric permittivity (up to $\sim 100$ ) in this direction. The spectral profile of the low-frequency mode clearly splits for temperatures below $\sim 200 \mathrm{~K}$. The frequency of these lowest phonons decreases with increasing atomic number of the rare-earth ion pointing towards an emerging instability of the langasite lattice. In Ho-LGS, a magneto-optical response is observed, where the lowest frequency phonon dynamics are seemingly effected by an applied magnetic field. This novel behavior could be evidence of hybridized spin-lattice dynamics, which may help explain the unusual magnetoelectric effect previously observed in this system. It may also shine light onto the perplexing spin-liquid behavior found in Nd-LGS.

\section{ACKNOWLEDGMENTS}

This work was supported by the Russian Science Foundation (16-12-10531) and by the Austrian Science Funds (W-1243, I 2816-N27, P 32404-N27).
[1] B. V. Mill and Y. V. Pisarevsky, Langasite-type materials: From discovery to present state, in Proceedings of the 2000 IEEE/EIA International Frequency Control Symposium and Exhibition (Cat. No. 00CH37052) (IEEE, Kansas City, MO, USA, 2000), pp. 133-144.
[2] K. Shimamura, H. Takeda, T. Kohno, and T. Fukuda, Growth and characterization of lanthanum gallium silicate $\mathrm{La}_{3} \mathrm{Ga}_{5} \mathrm{SiO}_{14}$ single crystals for piezoelectric applications, J. Cryst. Growth 163, 388 (1996). 
[3] J. Sato, H. Takeda, H. Morikoshi, K. Shimamura, P. Rudolph, and T. Fukuda, Czochralski growth of $\mathrm{RE}_{3} \mathrm{Ga}_{5} \mathrm{SiO}_{14}(\mathrm{RE}=\mathrm{La}$, $\mathrm{Pr}, \mathrm{Nd}$ ) single crystals for the analysis of the influence of rare earth substitution on piezoelectricity, J. Cryst. Growth 191, 746 (1998).

[4] N. Araki, H. Ohsato, K. Kakimoto, T. Kuribayashi, Y. Kudoh, and $\mathrm{H}$. Morikoshi, Origin of piezoelectricity for langasite $\mathrm{A}_{3}$ $\mathrm{Ga}_{5} \mathrm{SiO}_{14}(\mathrm{~A}=\mathrm{La}$ and $\mathrm{Nd})$ under high pressure, J. Eur. Ceram. Soc. 27, 4099 (2007).

[5] T. Iwataki, H. Ohsato, K. Tanaka, H. Morikoshi, J. Sato, and K. Kawasaki, Mechanism of the piezoelectricity of langasite based on the crystal structures, J. Eur. Ceram. Soc. 21, 1409 (2001).

[6] J. Detaint, J. Schwartzel, A. Zarka, B. Capelle, J. P. Denis, and E. Philippot, Bulk wave propagation and energy trapping in the new thermally compensated materials with trigonal symmetry, in Proceedings of IEEE 48th Annual Symposium on Frequency Control (IEEE, Boston, MA, USA, 1994), pp. 58-71.

[7] E. Ansorge, B. Schmidt, J. Sauerwald, and H. Fritze, Langasite for microelectromechanical systems, Phys. Status Solidi A 208, 377 (2011).

[8] P. Bordet, I. Gelard, K. Marty, A. Ibanez, J. Robert, V. Simonet, B. Canals, R. Ballou, and P. Lejay, Magnetic frustration on a Kagomé lattice in $\mathrm{R}_{3} \mathrm{Ga}_{5} \mathrm{SiO}_{14}$ langasites with $\mathrm{R}=\mathrm{Nd}$, $\mathrm{Pr}, \mathrm{J}$. Phys.: Condens. Matter 18, 5147 (2006).

[9] J. Robert, V. Simonet, B. Canals, R. Ballou, P. Bordet, P. Lejay, and A. Stunault, Spin-Liquid Correlations in the Nd-Langasite Anisotropic Kagomé Antiferromagnet, Phys. Rev. Lett. 96, 197205 (2006).

[10] H. D. Zhou, B. W. Vogt, J. A. Janik, Y.-J. Jo, L. Balicas, Y. Qiu, J. R. D. Copley, J. S. Gardner, and C. R. Wiebe, Partial Field-Induced Magnetic Order in the Spin-Liquid Kagomé $\mathrm{Nd}_{3} \mathrm{Ga}_{5} \mathrm{SiO}_{14}$, Phys. Rev. Lett. 99, 236401 (2007).

[11] V. Simonet, R. Ballou, J. Robert, B. Canals, F. Hippert, P. Bordet, P. Lejay, P. Fouquet, J. Ollivier, and D. Braithwaite, Hidden Magnetic Frustration by Quantum Relaxation in Anisotropic Nd Langasite, Phys. Rev. Lett. 100, 237204 (2008).

[12] K. Marty, V. Simonet, E. Ressouche, R. Ballou, P. Lejay, and P. Bordet, Single Domain Magnetic Helicity and Triangular Chirality in Structurally Enantiopure $\mathrm{Ba}_{3} \mathrm{NbFe}_{3} \mathrm{Si}_{2} \mathrm{O}_{14}$, Phys. Rev. Lett. 101, 247201 (2008).

[13] K. Marty, P. Bordet, V. Simonet, M. Loire, R. Ballou, C. Darie, J. Kljun, P. Bonville, O. Isnard, P. Lejay, B. Zawilski, and C. Simon, Magnetic and dielectric properties in the langasite-type compounds: $\mathrm{A}_{3} \mathrm{BFe}_{3} \mathrm{D}_{2} \mathrm{O}_{14}(\mathrm{~A}=\mathrm{Ba}, \mathrm{Sr}, \mathrm{Ca} ; \mathrm{B}=\mathrm{Ta}, \mathrm{Nb}, \mathrm{Sb} ; \mathrm{D}$ $=\mathrm{Ge}, \mathrm{Si})$, Phys. Rev. B 81, 054416 (2010).

[14] I. S. Lyubutin, P. G. Naumov, B. V. Mill, K. V. Frolov, and E. I. Demikhov, Structural and magnetic properties of the ironcontaining langasite family $\mathrm{A}_{3} \mathrm{MFe}_{3} \mathrm{X}_{2} \mathrm{O}_{14}(\mathrm{~A}=\mathrm{Ba}, \mathrm{Sr} ; \mathrm{M}=$ $\mathrm{Sb}, \mathrm{Nb}, \mathrm{Ta} ; \mathrm{X}=\mathrm{Si}, \mathrm{Ge}$ ) observed by Mössbauer spectroscopy, Phys. Rev. B 84, 214425 (2011).

[15] L. Chaix, R. Ballou, A. Cano, S. Petit, S. de Brion, J. Ollivier, L.-P. Regnault, E. Ressouche, E. Constable, C. V. Colin, A. Zorko, V. Scagnoli, J. Balay, P. Lejay, and V. Simonet, Helical bunching and symmetry lowering inducing multiferroicity in $\mathrm{Fe}$ langasites, Phys. Rev. B 93, 214419 (2016).

[16] L. Chaix, S. de Brion, F. Lévy-Bertrand, V. Simonet, R. Ballou, B. Canals, P. Lejay, J. B. Brubach, G. Creff, F. Willaert, P.
Roy, and A. Cano, THz Magnetoelectric Atomic Rotations in the Chiral Compound $\mathrm{Ba}_{3} \mathrm{NbFe}_{3} \mathrm{Si}_{2} \mathrm{O}_{14}$, Phys. Rev. Lett. 110, 157208 (2013).

[17] N. Lee, Y. J. Choi, and S.-W. Cheong, Magnetic control of ferroelectric polarization in a self-formed single magnetoelectric domain of multiferroic $\mathrm{Ba}_{3} \mathrm{NbFe}_{3} \mathrm{Si}_{2} \mathrm{O}_{14}$, Appl. Phys. Lett. 104, 072904 (2014).

[18] M. Ramakrishnan, E. Constable, A. Cano, M. Mostovoy, J. S. White, N. Gurung, E. Schierle, S. de Brion, C. V. Colin, F. Gay, P. Lejay, E. Ressouche, E. Weschke, V. Scagnoli, R. Ballou, V. Simonet, and U. Staub, Field-induced double spin spiral in a frustrated chiral magnet, npj Quantum Mater. 4, 60 (2019).

[19] L. Weymann, L. Bergen, T. Kain, A. Pimenov, A. Shuvaev, E. Constable, D. Szaller, B. V. Mill, A. M. Kuzmenko, V. Y. Ivanov, N. V. Kostyuchenko, A. I. Popov, A. K. Zvezdin, A. Pimenov, A. A. Mukhin, and M. Mostovoy, Unusual magnetoelectric effect in paramagnetic rare-earth langasite, npj Quantum Mater. 5, 61 (2020).

[20] A. B. Kuzmenko, Kramers-Kronig constrained variational analysis of optical spectra, Rev. Sci. Instrum. 76, 083108 (2005).

[21] G. Kozlov and A. Volkov, Millimeter and submillimeter wave spectroscopy of solids (Springer, Berlin, 1998) pp. 51-109.

[22] See Supplemental Material at http://link.aps.org/supplemental/ 10.1103/PhysRevB.104.024106 for broadband spectral results.

[23] N. Kuzmin, S. Klimin, B. Mavrin, K. Boldyrev, V. Chernyshev, B. Mill, and M. Popova, Lattice dynamics and structure of the new langasites $\mathrm{Ln}_{3} \mathrm{CrGe}_{3} \mathrm{Be}_{2} \mathrm{O}_{14}(\mathrm{Ln}=\mathrm{La}, \mathrm{Pr}, \mathrm{Nd})$ : Vibrational spectra and ab initio calculations, J. Phys. Chem. Solids 138, 109266 (2020).

[24] N. N. Kuzmin, S. A. Klimin, B. N. Mavrin, K. N. Boldyrev, V. A. Chernyshev, B. V. Mill, and M. N. Popova, Data on vibrational spectra of the langasites $\mathrm{Ln}_{3} \mathrm{CrGe}_{3} \mathrm{Be}_{2} \mathrm{O}_{14}(\mathrm{Ln}=$ La, Pr, Nd) and ab initio calculations, Data Brief 28, 104889 (2020).

[25] H. Usui, H. Kusakabe, M. Tokuda, K. Sguiyama, T. Hodhina, T. Tsurumi, and $\mathrm{H}$. Takeda, Structure and electrical properties of $\mathrm{Ba}_{3} \mathrm{TaGa}_{3} \mathrm{Si}_{2} \mathrm{O}_{14}$ single crystals grown by Czochralski method, J. Ceram. Soc. Jpn. 128, 441 (2020).

[26] B. A. Maksimov, V. N. Molchanov, B. V. Mill, E. L. Belokoneva, M. K. Rabadanov, A. A. Pugacheva, Y. V. Pisarevskii, and V. I. Simonov, Absolute structure of $\mathrm{La}_{3} \mathrm{Ga}_{5} \mathrm{SiO}_{14}$ langasite crystals, Crystallogr. Rep. 50, 751 (2005).

[27] C. Toulouse, M. Cazayous, S. de Brion, F. Levy-Bertrand, H. Barkaoui, P. Lejay, L. Chaix, M. B. Lepetit, J. B. Brubach, and P. Roy, Phonons in the multiferroic langasite $\mathrm{Ba}_{3} \mathrm{NbFe}_{3} \mathrm{Si}_{2} \mathrm{O}_{14}$ : Evidence for symmetry breaking, Phys. Rev. B 92, 104302 (2015).

[28] X. S. Xu, T. V. Brinzari, S. McGill, H. D. Zhou, C. R. Wiebe, and J. L. Musfeldt, Absence of Spin Liquid Behavior in $\mathrm{Nd}_{3} \mathrm{Ga}_{5} \mathrm{SiO}_{14}$ Using Magneto-Optical Spectroscopy, Phys. Rev. Lett. 103, 267402 (2009).

[29] E. Constable, R. Ballou, J. Robert, C. Decorse, J.-B. Brubach, P. Roy, E. Lhotel, L. Del-Rey, V. Simonet, S. Petit, and S. deBrion, Double vibronic process in the quantum spin ice candidate $\mathrm{Tb}_{2} \mathrm{Ti}_{2} \mathrm{O}_{7}$ revealed by terahertz spectroscopy, Phys. Rev. B 95, 020415(R) (2017). 